Pacific Journal of Mathematics

A RIESZ THEORY IN YON NEUMANN ALGEBRAS 


\title{
A RIESZ THEORY IN VON NEUMANN ALGEBRAS
}

\section{ANTON Ströh AND Johan Swart}

\begin{abstract}
An operator $T$ is called a Riesz operator relative to a von Neumann algebra $\mathscr{A}$ if $T-\lambda I$ is Fredholm relative to $\mathscr{A}$ for each $\lambda \neq 0$. Properties of Riesz operators are studied and a geometrical characterization of these operators are given. This characterization is used to show that a Riesz type of decomposition holds.
\end{abstract}

Introduction. The main theme of this paper is to introduce Riesz operators relative to a von Neumann algebra and to obtain a Riesz type of decomposition for these operators.

The theory of compact and Fredholm operators relative to a von Neumann algebra has been studied in detail by various authors (cf. [3], [4], [7], [8], [10], etc.). In the present paper Riesz operators are defined in a natural way via the Fredholm operators relative to a von Neumann algebra $\mathscr{A}$, i.e. $T$ will be called Riesz relative to $\mathscr{A}$ if $T-\lambda I$ is Fredholm relative to $\mathscr{A}$ for every $\lambda \neq 0$.

After some preliminaries in $\S 1$ we develop the basic results on Riesz operators in $\S 2$. These results are similar to results known for the classical case and will be used in the sequel. Section 3 contains a geometrical characterization of the Riesz operators. This may be considered as the main result of this paper, since it allows one to use the techniques of [4] and [5] to obtain the required Riesz decomposition in $\S 4$.

Whereas in the classical case the theory of Riesz operators has an intimate connection with spectral theory, it should be noted that in our representation we do not use spectral theory at all. Actually one cannot hope to obtain any results on the spectrum of a Riesz operator relative to a von Neumann algebra. In finite von Neumann algebras for instance all operators are Riesz. One can thus find Riesz operators with spectral properties very different from the classical case.

1. Preliminaries. Let $L(H)$ be the algebra of all bounded linear operators on a Hilbert space $H$. Throughout the paper $\mathscr{A}$ will denote a concrete von Neumann algebra on $H$. We denote by $\mathscr{F}$ the ideal generated by the projections which are finite relative to $\mathscr{A}$ (cf. [11], Chapter $\mathrm{V}$ for properties of the projection lattice $\mathscr{P}(\mathscr{A})$ on $\mathscr{A})$. The 
ideal of compact operators $\mathscr{K}$ relative to $\mathscr{A}$ is the uniform closure of $\mathscr{F}$. Let $\pi: \mathscr{A} \rightarrow \mathscr{A} / \mathscr{K}$ be the canonical quotient map. An operator $T \in \mathscr{A}$ is called a Fredholm operator (relative to $\mathscr{A}$ ) if $\pi(T)$ is invertible. For any $T \in \mathscr{A}$ we shall denote by $N_{T}$ the null projection and $R_{T}$ the range projection (cf. [3], (3.1) and (3.2)). It follows from [4], Theorem 1 and [7], Theorem 2.2 that $T$ is Fredholm iff $N_{T}$ is finite and $R_{T}$ is cofinite relative to $\mathscr{A}$ (cf. $\S 4$ for the definition of a cofinite range projection). The set of all Fredholm operators in $\mathscr{A}$ will be denoted by $\Phi$. We refer to [3] and [4] for the definition of the index function on $\Phi$ with values in a partially ordered abelian group. Let $\Phi_{0}$ denote the class of Fredholm operators $T$ with index zero (i.e. $N_{T} \sim N_{T^{*}}$ ).

For $T \in \mathscr{A}$ we denote the spectral radius by $r(T)$ and we shall call the spectrum of $\pi(T)$ in $\mathscr{A} / \mathscr{K}$ the (Wolf) essential spectrum of $T$ and denote it by $\sigma^{e}(T)$.

2. Riesz operators. An operator $T \in \mathscr{A}$ will be called a Riesz operator (relative to $\mathscr{A}$ ) if $\lambda I-T \in \Phi$ for every $\lambda \neq 0$. It is clear that $T$ is Riesz iff $\sigma^{e}(T)=\{0\}$, which is also equivalent to

$$
\lim _{n \rightarrow \infty}\left(\inf _{K \in \mathscr{K}}\left\|T^{n}-K\right\|\right)^{1 / n}=r(\pi(T))=0
$$

(cf. [7], 3.10). Since $\mathscr{F}$ is dense in $\mathscr{H}$ we may replace $\mathscr{K}$ with $\mathscr{F}$ in the last characterization. We shall denote the set of all Riesz operators by $\mathscr{R}$ and if the reference to $\mathscr{A}$ is necessary we denote this set by $\mathscr{R}(\mathscr{A})$.

REMARKS. 1. Since for a finite von Neumann algebra $\mathscr{A}$ we know that $\Phi=\mathscr{A}$ it is clear that then also $\mathscr{R}=\mathscr{A}$. The theory of Riesz operators in this case is trivial.

2. For any compact $K \in \mathscr{A}$ one has $r(\pi(K))=0$ from which it follows that $\mathscr{K} \subseteq \mathscr{R}$. There are many cases where this inclusion is strict.

3. In purely infinite von Neumann algebras the Riesz operators coincide with the quasinilpotent operators (recall that in this case $\mathscr{K}=$ $\{0\})$.

We denote by $[S, T]$ the commutator of $S$ and $T$, i.e. $[S, T] \equiv$ $S T-T S$. By using the well-known property that in any Banach algebra the relations $r(T S) \leq r(T) r(S)$ and $r(T+S) \leq r(T)+r(S)$ hold for any two commuting $S$ and $T$, one easily obtains the following proposition. 
2.1. Proposition. (a) If $S \in \mathscr{R}, T \in \mathscr{A}$ and $[S, T] \in \mathscr{K}$, then $S T, T S \in \mathscr{R}$.

(b) If $S, T \in \mathscr{R}$ and $[S, T] \in \mathscr{K}$ then $T+\alpha S \in \mathscr{R}$ for any $\alpha \in \mathbb{C}$.

(c) If a sequence $\left(T_{n}\right)$ of Riesz operators is uniformly convergent to $T \in \mathscr{A}$ and if $\left[T_{n}, T\right] \in \mathscr{K}$ for all $n \in \mathbb{N}$ then $T \in \mathscr{R}$.

It follows from 2.1 that the closed algebra generated by a Riesz operator is contained in $\mathscr{R}$.

2.2. Proposition. For $T \in \mathscr{A}$ we have that $T \in \mathscr{R}$ iff $T^{n} \in \mathscr{R}$ for any (and hence for all) $n \in \mathbb{N}$.

Proof. If $T \in \mathscr{R}$ then $T^{n} \in \mathscr{R}$ for any $n \in \mathbb{N}$ follows trivially from 2.1. Conversely if $T^{n} \in \mathscr{R}$ it follows by definition that

$$
\lim _{k \rightarrow \infty} \inf _{K \in \mathscr{K}}\left\|T^{n k}-K\right\|^{1 / n k}=0 \text {. }
$$

Since

$$
r(\pi(T))=\lim _{k \rightarrow \infty} \inf _{K \in \mathscr{K}}\left\|T^{k}-K\right\|^{1 / k}
$$

is finite one clearly has

$$
\lim _{k \rightarrow \infty} \inf _{K \in \mathscr{K}}\left\|T^{k}-K\right\|^{1 / k}=0
$$

From the fact that $\mathscr{K}$ is a two-sided ${ }^{*}$-ideal in $\mathscr{A}$ we have for any $T \in \mathscr{A}$ and $K \in \mathscr{K}$ that $r(\pi(T))=r\left(\pi\left(T^{*}\right)\right)$ and $r(\pi(T+K))=$ $r(\pi(T))$. Hence we obtain:

2.3. Proposition. (a) Let $T \in \mathscr{A}$ and $K \in \mathscr{K}$. Then $T \in \mathscr{R}$ iff $T^{*} \in \mathscr{R}$.

(b) $\mathscr{R}$ is stable under compact perturbations.

If $T$ is a normal operator in $\mathscr{A}$ it follows that $r(\pi(T))=\|\pi(T)\|_{\mathscr{A} / \mathscr{K}}$. Hence we have:

2.4. Proposition. For a normal operator $T \in \mathscr{R}$ iff $T \in \mathscr{K}$.

It seems that the following result is not known even for the classical case.

2.5. Proposition. If $T \in \mathscr{A}, S \in \mathscr{R}$ and $[S, T] \in \mathscr{K}$ then $\sigma^{e}(T+S)=\sigma^{e}(T)$. 
Proof. For any two commuting elements $a, b$ in a Banach algebra one knows that $\sigma(a+b) \subseteq \sigma(a)+\sigma(b)$, in particular $\sigma(\pi(T)+\pi(S)) \subseteq$ $\sigma(\pi(T))+\sigma(\pi(S))$. By assumption $\sigma(\pi(S))=\{0\}$. Hence $\sigma^{e}(T+S)$ $\subset \sigma^{e}(T)$. Similarly $\sigma^{e}(T)=\sigma^{e}(T+S-S) \subseteq \sigma^{e}(T+S)$.

The above-mentioned proposition may be used to prove a characterization of Riesz operators in von Neumann algebras which is similar to a result due to Schechter (cf. [9], Theorem 12).

2.6. Corollary. $T \in \mathscr{R}$ iff $T+S \in \Phi$ for all $S \in \Phi$ for which $[S, T] \in \mathscr{K}$.

Proof. Let $T \in \mathscr{R}$ and $S \in \Phi$ with the property that $[S, T] \in \mathscr{K}$, then we know that $0 \notin \sigma^{e}(S)=\sigma^{e}(T+S)$, so $T+S \in \Phi$. Since $[\lambda I, T]=0$ the converse is trivial.

For Riesz operators one obtains the following functional calculus.

2.7. Proposition. Let $f$ be a holomorphic function on an open set $U$ containing $\sigma(T)$ with $f(0)=0$. Then

(a) If $T \in \mathscr{R}$ then $f(T) \in \mathscr{R}$

(b) If $f(T) \in \mathscr{R}$ and $f$ does not vanish on $\sigma(T) \backslash\{0\}$ it follows that $T \in \mathscr{R}$.

Proof. (a) From our assumptions it follows that $f(T)=T g(T)$ where $g$ is holomorphic on $U$ and $[T, g(T)]=0$. Then (a) follows directly from $2.1(\mathrm{a})$.

(b) Since $\sigma^{e}(T) \subseteq \sigma(T)$ the functional calculus in $\mathscr{A} / \mathscr{K}$ shows that $\pi(f(T))=f(\pi(T))$, and by the spectral mapping theorem $f\left(\sigma^{e}(T)\right)=$ $\sigma^{e}(f(T))=\{0\}$. By hypothesis $f$ does not vanish on $\sigma(T) \backslash\{0\}$, leaving $\sigma^{e}(T)=\{0\}$ as the only possibility.

In any unital $\mathscr{C}^{*}$-algebra $\mathscr{A}$ it is known that $\sigma(x y) \backslash\{0\}=$ $\sigma(y x) \backslash\{0\}$ and $\sigma\left(u x u^{*}\right) \backslash\{0\}=\sigma(x) \backslash\{0\}$ hold for $x, y \in \mathscr{A}$ and $u \in \mathscr{A}$ unitary. The following proposition therefore follows:

2.8. Proposition. (a) $T S \in \mathscr{R}$ if and only if $S T \in \mathscr{R}$.

(b) If $S$ and $T$ are unitary equivalent, then $S \in \mathscr{R}$ iff $T \in \mathscr{R}$.

One can easily see from the next proposition that if a von Neumann algebra contains non-compact quasinilpotent operators, then $\mathscr{K}$ is properly contained in $\mathscr{R}$. 
2.9. Proposition. If $K \in \mathscr{K}$ and $Q \in \mathscr{A}$ is quasinilpotent, then $K+Q \in \mathscr{R}$.

Proof. This clearly follows from

$$
\left\|\pi(Q+K)^{n}\right\|^{1 / n}=\left\|\pi(Q)^{n}\right\|^{1 / n} \leq\left\|Q^{n}\right\|^{1 / n} \quad \text { for all } n \in \mathbb{N} .
$$

By the well-known West decomposition theorem (cf. [6], 3.33) the converse of 2.9 holds in the case where $\mathscr{A}=L(H)$. It is an open problem whether this is true in general von Neumann algebras. A partial converse can be obtained by using a result of Akemann and Pedersen [1]: If $T \in \mathscr{A}$ with $T^{n} \in \mathscr{K}$ for some $n \in \mathbb{N}$ (note that in this case $T \in \mathscr{R}$ by 2.2), then $T=K+Q$ where $K \in \mathscr{K}$ and $Q$ is nilpotent. This follows from the fact that [1] 4.3 implies that there exists a $K \in \mathscr{K}$ such that $(T-K)^{n}=0$.

2.10. Proposition (Generalized Fredholm alternative). Let $T \in$ $\mathscr{R}$. Then $(I-T) \in \Phi_{0}$.

Proof. By definition $(I-\lambda T) \in \Phi$ for all $\lambda \neq 0$. Since the index map on $\Phi$ is locally constant (cf. [4], Lemma 6), $\{I-\lambda T \mid \lambda \in[0,1]\}$ is contained in the same connected component of Fredholm operators and the result follows.

For any subset $B \subseteq \mathscr{A}$ we define the perturbation class of $B$ by

$$
P(B)=\{T \in \mathscr{A} \mid T+S \in B \text { for all } S \in B\} .
$$

In 2.3(b) we have seen that $\mathscr{K}$ is contained in the perturbation class of $\mathscr{R}$. The next proposition shows that one actually has equality:

2.11. Proposition. The perturbation class of $\mathscr{R}$ is the ideal $\mathscr{K}$ of compact operators.

Proof. Let $Q(\mathscr{A} / \mathscr{K})$ be the class of quasinilpotent elements of $\mathscr{A} / \mathscr{K}$. From a theorem due to Zemånek (cf. [2], BA2.8) we have:

$$
\begin{aligned}
\operatorname{rad}(\mathscr{A} / \mathscr{K}) & =\{\pi(T) \mid T \in \mathscr{A} \text { and } \pi(T)+Q(\mathscr{A} / \mathscr{K}) \subset Q(\mathscr{A} / \mathscr{K})\} \\
& =\{\pi(T) \mid T \in \mathscr{A} \text { and } T+S \in \mathscr{R} \text { for all } S \in \mathscr{R}\} .
\end{aligned}
$$

Since $\mathscr{A} / \mathscr{K}$ is a $\mathscr{C}^{*}$-algebra $\operatorname{rad}(\mathscr{A} / \mathscr{K})=\{0\}$, it then follows that

$$
\begin{aligned}
\mathscr{K} & =\pi^{-1}(\operatorname{rad}(\mathscr{A} \mid \mathscr{K})) \\
& =\{T \in \mathscr{A} \mid T+S \in \mathscr{R} \text { for all } S \in \mathscr{R}\} \\
& =P(\mathscr{R}) .
\end{aligned}
$$


2.12. THEOREM. The ideal $\mathscr{K}$ of compact operators is the largest two-sided ideal consisting of Riesz operators only.

Proof. We first show that every Riesz projection is finite. Let $E$ be a Riesz projection. Then $\lim _{n \rightarrow \infty}\left(\inf _{K \in \mathscr{K}}\|E-K\|\right)^{1 / n}=0$ and hence $E \in \mathscr{K}$. Since any compact projection is finite we have $E \in \mathscr{F}$. Let $\mathscr{T}$ be any two-sided ideal contained in $\mathscr{R}$. From the first part of the proof it then follows that $\overline{\mathscr{T}} \subset \overline{\mathscr{F}}=\mathscr{K}$.

In the last two results of this section we show that the class of Riesz operators behaves well under reduction with respect to central projections as well as under decompositions of the von Neumann algebra. These results will be needed later in an important counterexample.

Similar results for the class of compact operators in $\mathscr{A}$ were obtained by Kaftal (cf. [8], 2.1, 2.2).

Let $E$ be a central projection in the von Neumann algebra $\mathscr{A}$. We shall then use the following notation: $\mathscr{A}_{E}:=\mathscr{A} E$ and $\mathscr{R}_{E}:=\mathscr{R} E$.

2.13. LEMMA. With the above notation one has that $\mathscr{R}_{E}=\mathscr{R}\left(\mathscr{A}_{E}\right)$.

Proof. Let $T \in \mathscr{R}_{E}$ and $\lambda \neq 0$ be given. There exists an $S \in \mathscr{R}$ such that $T=S E$. Then $S_{\lambda}:=\lambda I-S$ is invertible modulo $\mathscr{K}$, i.e. there exists an $S_{\lambda}^{\prime}$ such that $S_{\lambda} S_{\lambda}^{\prime} \in I+\mathscr{K}$ and $S_{\lambda}^{\prime} S_{\lambda} \in I+\mathscr{K}$ hence $E S_{\lambda} E S_{\lambda}^{\prime} \in E+\mathscr{K}_{E}$ and $E S_{\lambda}^{\prime} E S_{\lambda} \in E+\mathscr{K}_{E}$. We know that $\mathscr{K}\left(\mathscr{A}_{E}\right)=\mathscr{K}_{E}$ and therefore $\lambda E-T\left(=E S_{\lambda}\right)$ is invertible modulo $\mathscr{K}\left(\mathscr{A}_{E}\right)$. Hence $T \in \mathscr{R}\left(\mathscr{A}_{E}\right)$. Conversely, suppose $T \in \mathscr{R}\left(\mathscr{A}_{E}\right)$ and $\lambda \neq 0$. Then $S_{\lambda}:=E-1 / \lambda T \in \Phi\left(\mathscr{A}_{E}\right)$. Thus there exists $S_{\lambda}^{\prime} \in \mathscr{A}_{E}$ such that

$$
S_{\lambda} S_{\lambda}^{\prime} \in E+\mathscr{K}_{E} \quad \text { and } \quad S_{\lambda}^{\prime} S_{\lambda} \in E+\mathscr{K}_{E} .
$$

Let $A_{\lambda}=S_{\lambda}+I-E$ and $B_{\lambda}=S_{\lambda}^{\prime}+I-E$. Then $A_{\lambda}, B_{\lambda} \in \mathscr{A}$ and

$$
A_{\lambda} B_{\lambda} \in I+\mathscr{K} \quad \text { and } \quad B_{\lambda} A_{\lambda} \in I+\mathscr{K} \text {. }
$$

Thus $I=1 / \lambda T=A_{\lambda} \in \Phi(\mathscr{A})$ for all $\lambda \neq 0$ and therefore $T \in \mathscr{R}$ and since $T=T E$, we have that $T \in \mathscr{R}_{E}$.

Let $\mathscr{A}=\sum_{i \in I}^{\oplus} \mathscr{A}_{i}$ be the direct sum of von Neumann algebras $\mathscr{A}_{i}$. We may identify the identity of $\mathscr{A}_{i}$ with a central projection $E_{i} \in \mathscr{A}$ and $\mathscr{A}_{i}$ with $\mathscr{A} E_{i}$. Denote $\left\{T \in \mathscr{A}: T E_{i} \in \mathscr{R}\left(\mathscr{A}_{i}\right)\right\}$ by $\sum_{i \in I}^{\oplus} \mathscr{R}\left(\mathscr{A}_{i}\right)$.

For $L \subseteq I$ we may identify $\sum_{i \in L}^{\oplus} \mathscr{A}_{i}$ with a closed subalgebra of $\sum_{i \in I}^{\oplus} \mathscr{A}_{i}$ in an obvious way. 
2.14. Proposition. Let $\mathscr{A}=\sum_{i \in I}^{\oplus} \mathscr{A}_{i}$. Then $\mathscr{R}(\mathscr{A}) \subseteq \sum_{i \in I}^{\oplus} \mathscr{R}\left(\mathscr{A}_{i}\right)$ and equality holds if at most finitely many $E_{i}$ are infinite.

Proof. The inclusion follows directly by application of the previous lemma. Suppose then that $E_{i}$ is finite for all $i \notin J$, where $J$ is some finite subset $J \subseteq I$. Let $T \in \sum_{i \in I}^{\oplus} \mathscr{R}\left(\mathscr{A}_{i}\right)$ and $\lambda \neq 0$. Then if $T=\sum_{i \in I}^{\oplus} T_{i}, S_{i, \lambda}:=E_{i}-1 / \lambda T_{i} \in \Phi\left(\mathscr{A}_{i}\right)$ from which it follows that there exist $S_{i, \lambda}^{\prime}$ and $K_{i, \lambda}, K_{i, \lambda}^{\prime} \in \mathscr{K}\left(\mathscr{A}_{i}\right)$ such that

$S_{i, \lambda} S_{i, \lambda}^{\prime}=E_{i}+K_{i, \lambda} \quad$ and $\quad S_{i, \lambda}^{\prime} S_{i, \lambda}=E_{i}+K_{i, \lambda}^{\prime} \quad$ for every $i \in I$.

For $i \notin J$ we may choose $S_{i, \lambda}^{\prime}=0$ and $K_{i, \lambda}^{\prime}=-E_{i}$.

Let $S_{\lambda}=\sum_{i \in I}^{\oplus} S_{i, \lambda}$ and $S_{\lambda}^{\prime}=\sum_{i \in I}^{\oplus} S_{i, \lambda}^{\prime}$. The last series is an element of $\mathscr{A}$ since it actually reduces to a finite sum by our choice of $S_{i, \lambda}^{\prime}$.

Then clearly, $S_{\lambda} S_{\lambda}^{\prime} \in I+\sum_{i \in I}^{\oplus} \mathscr{K}\left(\mathscr{A}_{i}\right)=I+\mathscr{K}(\mathscr{A})$ (cf. [8], 2.2) and similarly $S_{\lambda}^{\prime} S_{\lambda} \in I+\mathscr{K}(\mathscr{A})$. Since $S_{\lambda}=I-1 / \lambda T$ it follows that $T \in \mathscr{R}(\mathscr{A})$.

3. Characterizations of Riesz operators relative to a von Neumann algebra. Smyth obtained a geometric characterization for Riesz operators on a general Banach space (cf. [2], 0.3.5). In proving this result a somewhat laborious machinery of vector sequence spaces was needed. We shall prove a similar result for general von Neumann algebras which gives an elegant proof of Smyth's result for the $L(H)$-case.

For an operator $T$ in a von Neumann algebra $\mathscr{A}$ the following property (referred to as property A) will be used to characterize the Riesz operators relative to $\mathscr{A}$.

A. For every $\varepsilon>0$ there exists an $n \in \mathbb{N}$, a finite projection $P_{\varepsilon} \in \mathscr{P}(\mathscr{A})$ and a bounded set $N_{\varepsilon} \subseteq P_{\varepsilon}(H)$ such that for each $x \in U_{H}$ there exists a $y \in N_{\varepsilon}$ such that $\left\|T^{n} x-y\right\|<\varepsilon^{n}$. (Here and in the following $U_{H}$ will denote the unit ball of $H$.)

3.1. Lemma. If $T \in \mathscr{A}$ has property $\mathrm{A}$ then $T^{m}$ also has property A for all $m \in \mathbb{N}$.

Proof. Without loss of generality we may assume $T^{m} \neq 0$. For $\varepsilon>0$, put $\delta=\varepsilon /\left\|T^{m-1}\right\|$. By assumption there exist an $n \in \mathbb{N}$, a finite projection $P_{\delta}$ and a bounded set $N_{\delta} \subseteq P_{\delta}(H)$ such that for each $w \in U_{H}$ there exists a $z \in N_{\delta}$ such that $\left\|T^{n} w-z\right\|<\delta^{n}$. Let $N_{\varepsilon}=\left\|T^{m-1}\right\|^{n} N_{\delta}$ and $P_{\varepsilon}=P_{\delta}$. Then for $x \in U_{H}$ it follows that $\left\|T^{m n} x-y\right\|<\varepsilon^{n}$ for some $y \in N_{\varepsilon}$. 
3.2. Theorem. Let $T \in \mathscr{A}$. Then $T \in \mathscr{R}$ if and only if $T$ has property A.

Proof. Let $T \in \mathscr{R}$ and $\varepsilon>0$. Then since

$$
\lim _{n \rightarrow \infty}\left(\inf _{F \in \mathscr{F}}\left\|T^{n}-F\right\|\right)^{1 / n}=0
$$

there is an $n \in \mathbb{N}$ and an $F_{\varepsilon} \in \mathscr{F}$ such that

$$
\left\|T^{n}-F_{\varepsilon}\right\|<\varepsilon^{n} \text {. }
$$

Let $P_{\varepsilon}=R_{F_{\varepsilon}}$ and $N_{\varepsilon}=F_{\varepsilon}\left(U_{H}\right)$, then $P_{\varepsilon}$ is a finite projection and $N_{\varepsilon}$ is a bounded subset of $P_{\varepsilon}(H)$. By (1) $\left\|T^{n} x-F_{\varepsilon} x\right\|<\varepsilon^{n}$, for all $x \in U_{H}$. This proves property $\mathrm{A}$.

Conversely let $T$ have property A. We are going to show that there exists a subsequence of $\left\{\left(\inf _{K \in \mathscr{K}}\left\|T^{n}-K\right\|\right)^{1 / n}\right\}_{n}$ which converges to zero, implying that the spectral radius of $\pi(T)$ vanishes.

Let $\varepsilon>0$. Then there exist an $n \in \mathbb{N}$, a finite projection $P_{\varepsilon} \in$ $\mathscr{P}(\mathscr{A})$ and a bounded set $N_{\varepsilon} \subseteq P_{\varepsilon}(H)$ such that for every $x \in U_{H}$ there exists a $y \in N_{\varepsilon}$ such that

$$
\left\|T^{n} x-y\right\|<\varepsilon^{n} .
$$

Thus $\left\|T^{n} x-P_{\varepsilon} T^{n} x\right\|=\inf _{w \in P_{\varepsilon}(H)}\left\|T^{n} x-w\right\|<\varepsilon^{n}$. This holds for every $x \in U_{H}$, hence

$$
\left\|T^{n}-P_{\varepsilon} T^{n}\right\| \leq \varepsilon^{n} .
$$

Since $P_{\varepsilon}$ is finite and therefore $P_{\varepsilon} T^{n} \in \mathscr{K}(\mathscr{A})$, it follows that for any $\varepsilon>0$ there exists an $n \in \mathbb{N}$ such that $\left(\inf _{K \in \mathscr{K}}\left\|T^{n}-K\right\|\right)^{1 / n} \leq \varepsilon$.

We now find the zero converging subsequence recursively. There exists an $n_{1} \in \mathbb{N}$ such that $\left(\inf _{K \in \mathscr{K}}\left\|T^{n_{2}}-K\right\|\right)^{1 / n_{1}}<1$. Since $T^{n_{1}+1}$ has property A by 3.1, there exists an $m_{1} \in \mathbb{N}$ such that

$$
\left(\inf _{K \in \mathscr{K}}\left\|\left(T^{n_{1}+1}\right)^{m_{1}}-K\right\|\right)^{1 / m_{1}}<(1 / 2)^{n_{1}+1} .
$$

Let $n_{2}=\left(n_{1}+1\right) m_{1}$. Then clearly $n_{1}<n_{2}$ and from (2) it follows that

$$
\left(\inf _{K \in \mathscr{K}}\left\|T^{n_{2}}-K\right\|\right)^{1 / n_{2}}<1 / 2
$$

Repeating this argument one finds a monotone increasing sequence of positive integers $n_{1}<n_{2}<\cdots<n_{k}<\cdots$ such that

$$
\left(\inf _{K \in \mathscr{K}}\left\|T^{n_{k}}-K\right\|\right)^{1 / n_{k}}<1 / k \quad \text { for every } k \in \mathbb{N} \text {. }
$$


REMARK. It should be noted that in the case where $\mathscr{A}=L(H)$, property A coincides with the notion of a finite $\varepsilon^{n}$-net for $T^{n}\left(U_{H}\right)$ (cf. [2], $\S 0.3$ for the definition of an $\varepsilon$-net).

From the proof of 3.2 we have:

3.3. COROLlaRY. $T \in \mathscr{R}$ if and only if for every $\varepsilon>0$ there exist an $n \in \mathbb{N}$ and a projection $Q \in \mathscr{P}(\mathscr{A})$ such that $\left\|Q T^{n}\right\| \leq \varepsilon^{n}$ and $I-Q$ is finite.

Proof. If $T \in \mathscr{R}$ it has property A. Now if we put $Q=I-P_{\varepsilon}$ in the converse part of the proof of 3.2 the condition holds. Clearly the condition implies property $\mathrm{A}$ and the result follows.

3.4. Corollary. Let $S, T \in \mathscr{A}$ be commuting. If $T \in \mathscr{R}$ and $S(H) \subseteq T(H)$ then $S \in \mathscr{R}$.

Proof. Let $\varepsilon>0$ be given. Under the conditions of the theorem there exists an $\alpha>0$ such that for any $n \in \mathbb{N}$ one has

$$
S^{n}\left(U_{H}\right) \subseteq \alpha^{n} \overline{T^{n}\left(U_{H}\right)}
$$

(cf. [2], 0.4.1, 0.4.3).

Since $T \in \mathscr{R}$ there exist an $n \in \mathbb{N}$, a finite projection $P_{\varepsilon} \in \mathscr{P}(\mathscr{A})$ and a bounded set $N_{\varepsilon} \subseteq P_{\varepsilon}(H)$ such that for each $x \in U_{H}$ there exists a $y \in N_{\varepsilon}$ with

$$
\left\|T^{n} x-y\right\|<(\varepsilon / 2 \alpha)^{n} .
$$

Let $x \in U_{H}$, then it follows from (1) that there exists a $z \in U_{H}$ such that $\left\|S^{n} x-\alpha^{n} T^{n} z\right\|<\varepsilon^{n} / 2$.

$\operatorname{By}(2)$ there exists a $w \in N_{\varepsilon}$ such that $\left\|\alpha^{n} T^{n} z-\alpha^{n} w\right\|<\varepsilon^{n} / 2^{n}$. Thus $\left\|S^{n} x-\alpha^{n} w\right\|<\varepsilon^{n}$. By noting that the set $\alpha^{n} N_{\varepsilon} \subset P_{\varepsilon}(H)$ is bounded the corollary follows.

4. Riesz decomposition. In [5] a Riesz type of decomposition was obtained for compact operators in a von Neumann algebra. With our characterization 3.2 and its Corollary 3.3 in hand we can now use the techniques of [3] and [5] to obtain a Riesz type of decomposition for Riesz operators. It should be noted that all proofs are similar to the proofs in [3], [5]. Hence we shall only give attention to the essential differences.

For $T \in \mathscr{A}$ let

$$
\begin{array}{lll}
N_{n}:=N_{(I-T)^{n}} ; & F_{n}:=N_{n+1}-N_{n}, & n=0,1,2, \ldots, \\
R_{n}:=R_{(I-T)^{n}} ; & G_{n}:=R_{n}-R_{n+1}, & n=0,1,2, \ldots
\end{array}
$$


Note that $\left(N_{n}\right)$ is non-decreasing (i.e. $N_{n+r} N_{n}=N_{n}$ for all $r \in \mathbb{N}$ ) and $\left(R_{n}\right)$ is non-increasing (i.e. $R_{n} R_{n+r}=R_{n+r}$ for all $r \in \mathbb{N}$ ).

The range projection $R_{T}$ will be called (relatively) cofinite if $I-R_{T}$ is finite and if there exists a projection $Q \in \mathscr{A}$ such that $Q(H) \subseteq$ $T(H)$ with $R_{T}-Q$ finite. In $L(H)$ this coincides with the classical definition of cofiniteness.

\subsection{LEMMA. With the above notation}

(a) $N_{n+r} T^{k} N_{n}=T^{k} N_{n}$,

(b) $F_{n} T^{k} F_{n}=F_{n}$,

(c) $R_{n} T^{k} R_{n+r}=T^{k} R_{n+r}$,

(d) $G_{n} T^{k} G_{n}=G_{n}$,

for $n=0,1,2, \ldots ; r=0,1, \ldots ; k=1,2, \ldots$.

Proof. (a) and (b) follow by induction (on $k$ ) and by using the relation $\left(I-N_{n}\right) T N_{n+1}=F_{n}$ which follows from the properties of the projection $N_{n}$ (cf. [3]). Similarly (c) and (d) follow by using the relation $\left(I-R_{n+1}\right) T R_{n}=G_{n}$ which follows from the properties of the range projection.

4.2. TheOREM. Let $T \in \mathscr{R}$. Then the following hold:

(a) $N_{n}$ is relatively finite and $R_{n}$ relatively cofinite.

(b) If $N_{\infty}=\sup _{n \in \mathbb{N}} N_{n}$ and $R_{\infty}=\inf _{n \in \mathbb{N}} R_{n}$ then both $N_{\infty}(H)$ and $R_{\infty}(H)$ are invariant under $T^{k}$ for any $k \in \mathbb{N}$.

(c) $N_{\infty}$ is relatively finite and $N_{\infty} \sim I-R_{\infty}$.

(d) $\inf \left\{N_{\infty}, R_{\infty}\right\}=0$ and $\sup \left\{N_{\infty}, R_{\infty}\right\}=I$.

Proof. (a) Clearly $(I-T) \in \Phi$. By ([7], 2.2) $N_{1}$ is relatively finite and $R_{1}$ is relatively cofinite. For $n \in \mathbb{N}, n>1$ it follows from 2.1 that $(I-T)^{n}=I-T_{0}$ where $T_{0} \in \mathscr{R}$ and as before it follows that $N_{n}$ is relatively finite and $R_{n}$ relatively cofinite.

(b) This follows from 4.1(a) and (c) for $r=0$ and taking the strong operator limit on both sides.

(c) By using 3.3 and 4.1 the proof for the relative finiteness of $N_{\infty}$ for the compact case may be carried over virtually word for word by only replacing $T$ with $T^{n}$. From 2.10 and the fact that $\Phi_{0}$ is a semigroup, it follows that $N_{n} \sim I-R_{n}$ and hence $N_{\infty} \sim I-R_{\infty}$ follows similarly as for the compact case, cf. [3], Theorem 2, (i).

(d) This again follows along the lines of [3], Theorem 2 (iv) and [5], Theorem 3 (ii) by only noting that $(I-T)^{k}=I-T_{(k)}$ where $T_{(k)} \in \mathscr{R}$. 
It is well-known that both the sequences $\left(N_{n}\right)$ and $\left(R_{n}\right)$ eventually become stationary in the classical case. The following example shows that this is not always the case in general von Neumann algebras.

EXAMPLE. Let

$$
\mathscr{A}=\sum_{n=1}^{\infty} L\left(H_{n}\right)
$$

where $H_{n}=H$ is a separable Hilbert space. Let $T_{k} \in L(H)$ be defined by

$$
T_{k}\left(\sum_{i=1}^{\infty} x_{i} \phi_{i}\right)=x_{1} \phi_{1}+\sum_{i=2}^{k+1}\left(x_{i}-x_{i-1}\right) \phi_{i},
$$

where $\left\{\phi_{i} \mid i \in \mathbb{N}\right\}$ is any orthonormal basis for $H$. It is easy to see that

$$
N\left(I-T_{k}\right) \neq N\left(I-T_{k}\right)^{2} \neq \cdots \neq N\left(I-T_{k}\right)^{k+1}=N\left(I-T_{k}\right)^{k+r}
$$

for all $k, r \in \mathbb{N}$. Let

$$
\bar{I}=\sum_{n=1}^{\infty} I_{n} \quad \text { where } I_{n}=I \text { for all } n \in \mathbb{N} \text { and } T:=\sum_{n=1}^{\infty} T_{n} .
$$

Then $T$ is compact and hence Riesz relative to $\mathscr{A}$. However

$$
N(\bar{I}-T)^{k} \varsubsetneqq N(\bar{I}-T)^{k+r} \quad \text { for all } k, r \in \mathbb{N} \text {. }
$$

\section{REFERENCES}

[1] C. A. Akemann and G. K. Pedersen, Ideal perturbations of elements in $\mathscr{C}^{*}$ algebras, Math. Scand., 41 (1977), 117-139.

[2] B. A. Barnes, G. J. Murphy, M. R. F. Smyth and T. T. West, Riesz and Fredholm Theory in Banach Algebras, Pitman Adv. Publ. Program, London; 1982.

[3] M. Breuer, Fredholm theories in von Neumann algebras I, Math. Ann., 178 (1968), 243-254.

[4] $\quad$ Fredholm theories in von Neumann algebras II, Math. Ann., 180 (1969), 313-325.

[5] M. Breuer and R. S. Butcher, A generalized Riesz-Schauder decomposition theorem, Math. Ann., 203 (1973), 211-230.

[6] H. R. Dowson, Spectral Theory of Linear Operators, Academic Press, New York, 1978.

[7] V. Kaftal, On the theory of compact operators in von Neumann algebras I, Indiana Univ. Math. J., 26 (1977), 447-457.

[8] _ Almost Fredholm operators in von Neumann algebras, Integral Equations Operator Theory, 5 (1982), 50-70. 
[9] M. Schechter, Riesz operators and Fredholm perturbations, Bull. Amer. Math. Soc., 74 (1968), 1139-1144.

[10] M. G. Sonis, On a class of operators in von Neumann algebras with Segal measure on the projectors, Math. USSR Sbornik, 13 (1971), 344-359.

[11] M. Takesaki, Theory of Operator Algebras I, Springer, New York, 1979.

[12] F. B. Wright, A reduction for algebras of finite type, Ann. of Math., 60 (1954), 560-570.

Received October 15, 1988.

UNIVERSITY OF PRETORIA

0002 PRETORIA

REPUblic OF SOUTH AFrica 


\section{PACIFIC JOURNAL OF MATHEMATICS EDITORS}

\author{
V. S. VARADARAJAN \\ (Managing Editor) \\ University of California \\ Los Angeles, CA 90024-1555-05 \\ Herbert Clemens \\ University of Utah \\ Salt Lake City, UT 84112 \\ THOMAS ENRIGHT \\ University of California, San Diego \\ La Jolla, CA 92093
}

R. FINN

Stanford University

Stanford, CA 94305

HeRmanN FlaschKa

University of Arizona

Tucson, AZ 85721

VAUGHAN F. R. JONES

University of California

Berkeley, CA 94720

STEVEN KERCKHOFF

Stanford University

Stanford, CA 94305
C. C. MOORE

University of California

Berkeley, CA 94720

Martin ScharlemanN

University of California

Santa Barbara, CA 93106

\section{HAROLD STARK}

University of California, San Diego La Jolla, CA 92093

\section{ASSOCIATE EDITORS}
R. ARENS
E. F. BECKENBACH
B. H. NeumanN
F. WolF
K. YoshidA
(1906-1982)

(1904-1989)
TIONS

UNIVERSITY OF ARIZONA

UNIVERSITY OF BRITISH COLUMBIA

CALIFORNIA INSTITUTE OF TECHNOLOGY

UNIVERSITY OF CALIFORNIA

MONTANA STATE UNIVERSITY

UNIVERSITY OF NEVADA, RENO

NEW MEXICO STATE UNIVERSITY

OREGON STATE UNIVERSITY
UNIVERSITY OF OREGON

UNIVERSITY OF SOUTHERN CALIFORNIA

STANFORD UNIVERSITY

UNIVERSITY OF HAWAII

UNIVERSITY OF TOKYO

UNIVERSITY OF UTAH

WASHINGTON STATE UNIVERSITY

UNIVERSITY OF WASHINGTON 


\section{Pacific Journal of Mathematics}

\section{Vol. 148, No. $1 \quad$ March, 1991}

David Marion Arnold and Charles Irvin Vinsonhaler, Duality and

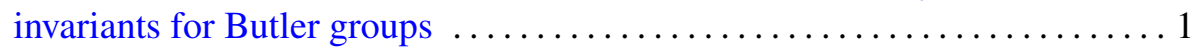

Philippe Delanoë, Obstruction to prescribed positive Ricci curvature . . . . . 11

María J. Druetta, Nonpositively curved homogeneous spaces of dimension

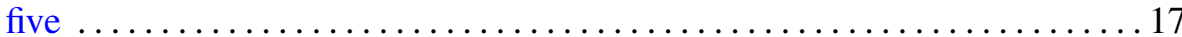

Robert Fitzgerald, Combinatorial techniques and abstract Witt rings III .... 39

Maria Girardi, Dentability, trees, and Dunford-Pettis operators on $L_{1} \ldots \ldots 59$

Krzysztof Jarosz, Ultraproducts and small bound perturbations $\ldots \ldots \ldots \ldots 81$

Russell David Lyons, The local structure of some measure-algebra homomorphisms .................................. 89

Fiona Anne Murnaghan, Asymptotic behaviour of supercuspidal characters of $p$-adic $\mathrm{GL}_{3}$ and $\mathrm{GL}_{4}$ : the generic unramified case $\ldots \ldots \ldots \ldots \ldots 107$

H. Rouhani, Quasi-rotation $C^{*}$-algebras $\ldots \ldots \ldots \ldots \ldots \ldots \ldots \ldots \ldots \ldots \ldots \ldots \ldots \ldots$

Ignacio Sols Lucía, Michał Szurek and Jaroslaw Wisniewski, Rank-2

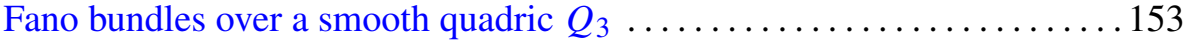

Martin Strake and Gerard Walschap, Ricci curvature and volume

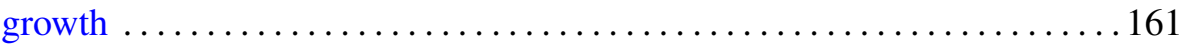

Anton Ströh and Johan Swart, A Riesz theory in von Neumann algebras . . 169

Ming Wang, The classification of flat compact complete space-forms with metric of signature $(2,2)$ 